\title{
Dengue and SARS-CoV-2 Co-Circulation Early Warning according to Climate Variations in Cuba
}

\author{
Linares Vega $\mathrm{Y}^{1 *}$ and Ortiz Bulto $\mathrm{PL}^{2}$ \\ ${ }^{1}$ Provincial Meteorological Center, Meteorological Institute, Cuba \\ ${ }^{2}$ Climate Center, Meteorological Institute, Cuba
}

*Corresponding author: Yazenia Linares Vega, Provincial Meteorological Center, Meteorological Institute, Cuba, Tel: 5353768226, Email: lyazenia@gmail.com

\section{Short Communication}

Volume 4 Issue 2

Received Date: September 03, 2021

Published Date: September 24, 2021

DOI: $10.23880 /$ oajmms-16000146

\section{Abstract}

Dengue and Severe Acute Respiratory Syndromes Coronavirus 2 (SARS-CoV-2) co-circulation is a reality resulting to be more dangerous in regions that are endemic for arboviruses. Circulation of both viruses continue spreading, mainly in tropical settings causing a high impact on the health systems of the countries. Therefore, forecasting the circulation of SARS-CoV-2 virus and dengue from the climatic variability a temporal and spatial scale allows to perfect the warning system and provides a tool for decision makers in the health system to take the necessary control measures.

Methods: Ecological study with retrospective-prospective analysis of the series of the SARS- CoV-2 viruses, dengue, as well as, Aedes focus with their index and the climatic anomalies described by the complex climatic Index of Bulto $\left(\mathrm{BI}_{1, \mathrm{r}, \mathrm{t}}\right.$ ). Interpolation method for the spatial structure with continuous information of 1200 nodes (Raster format) was generated. Kriging method combined with the method of the inverse distance (IDM) was implemented with a resolution of $10 \mathrm{~km}^{2}$. The Multivariate Moran Index was used to determine the spatial correlation. For the prediction, the Simultaneous Autoregressive Models and the Spatial Autoregressive Conditional Models were used. Temporal modeling was carried out by using the Heteroscedastic Conditional Autoregressive and Autoregressive Models, both with exogenous variables. To obtain the co-circulation risk forecast maps, a stratification of the circulation of both viruses is carried out and map algebra is applied.

Results: The moments of greatest risk Dengue and SARS-CoV-2 co-circulation are the months corresponding to the second quarter of the rainy period August-October with very humid conditions, very high temperatures, high volume of precipitation, high cloudiness, being potential predictors. All these results led to the creation of the dengue alert system and co-circulation with SARS-CoV-2 based on the observation and forecast of climatic factors. The areas with major viral co-circulation were the Central-Eastern region.

Conclusions: It is evident that the climate is an important determinant for the health sector, and in particular to understand the Dengue and SARS-CoV-2 behavior and co-circulation, which strengthens the active health surveillance system.

Keywords: Climate; Co-Circulation; Dengue; SARS-CoV-2; Early Warning System

Abbreviations: INSMET: Institute of Meteorology; IPK: Pedro Kouri Institute of Tropical Medicine; MINSAP: Ministry of Public Health; RSV: Respiratory Syncytial Virus; ARI: Acute Respiratory Infections; BI: Bulto Index; IDM: Inverse Distance.

\section{Introduction}

Increasing anomalies in climate variability, whether local or global, natural or anthropogenic, affect the functioning of host/vector systems and may have repercussions on their 


\section{Open Access Journal of Mycology \& Mycological Sciences}

extinction or survival, on their spread and on the emergence of diseases. Scientific evidence already shows that this change influences the increase and distribution of infectious diseases of medical importance [1,2].

In order carry out the studies of climate variability and change on human health, Cuba has created a working network made up of researchers from the Institute of Meteorology (INSMET) belonging to the Ministry of Science, Technology and Environment (CITMA) and the Pedro Kouri Institute of Tropical Medicine (IPK) belonging to the Ministry of Public Health (MINSAP). This climate-health group develops research aimed at deepening the response mechanisms of viruses to a changing climate, including Dengue and SARS$\mathrm{CoV}-2$. The group works from a methodological procedure with a complex approach for studies of variability and climate change. This modelling methodology has been used in other countries with a tropical climate (Panama, Costa Rica, Salvador Guatemala Nicaragua, Honduras, Dominican Republic, among others), as well as for infectious diseases $[1,3-8]$.

\section{Climate Effect on Dengue and Aedes Aegypti}

Climate is an important determinant of the temporalspatial distribution of Dengue. It is currently the main viral disease transmitted by Aedes Aegypti vector in terms of morbidity and mortality. Geographic spread and magnitude of the disease in recent decades has paralleled rapid population growth, unplanned urbanization, and the unprecedented increase in population mobility [9].

It is known that high temperatures increase the development speed of the larvae and consequently of the adult mosquito and can lead to an increase in the rate of bite and replication of the virus in the mosquito. Variability in rainfall influences the availability of vector breeding sites and consequently its abundance. Humidity influences vector half-life and potentially transmission. Some studies suggest that humidity is an important climatic predictor in the global occurrence of Dengue. However, being climatic conditions favorable for Ae aegypti, is not enough criterion in the transmission and dissemination of [10].

The mathematical models used in Dengue studies try to quantify the contribution of the virus, the host, the vector and the ecological factors in the transmission dynamics. This is generally not linear, showing strong seasonality, multi-year oscillations and non- stationary temporal variations, that in turn can vary in time and space. These models have tried, on the one hand, to explain the biological and ecological mechanisms that influence transmission dynamics and, on the other, to make predictions of the future magnitude, time and place of transmission [2,10-12].

\section{SARS-CoV-2 and its Relationship with the Climate}

The growing expansion of microorganisms such as viruses is not only due to anthropic pressures, because of human movements towards virgin natural spaces, for the purpose of urbanization and obtaining food or natural resources that cause deforestation. It is also produced by the pressure that climatic variations and changes cause on ecosystems, which modify natural habitats.

Climatic variability is an important determinant in the temporal-spatial distribution of respiratory viruses, which leads to them being more resistant, by reinforcing their power of mutability and adaptation to new conditions that results in the increase of viral diseases. It is estimated that $90 \%$ of respiratory infections are related to viruses. Influenza virus, Respiratory Syncytial Virus (RSV), and the new SARS-CoV-2 coronavirus cause the highest morbidity and mortality rates, mainly in young children, immunosuppressed patients, and the elderly [7].

The emergence of the new SARS-CoV-2 coronavirus, its rapid and wide spread and different variants, is causing the largest pandemic in history both in expansion and duration in time, claiming thousands of lives due to the high fatality rate and causing a significant burden on Acute Respiratory Infections (ARI). Until august 31, more than 190 countries in the world with COVID-19 cases are reported, with 218,511,670 confirmed cases and 4,532,508 deaths for a fatality of $2.07 \%$. In the Americas region, $85,038,849$ confirmed cases are reported, $38.92 \%$ of the total cases reported in the world, 2,125,437 deaths for a fatality of $2.5 \%$. Cuba accumulates 7,985,117 samples carried out and $6,59,464$ positives, with 5377 deaths, for a fatality of $0.82 \%$, a figure that is very low compared to the world and with the Americas, despite being a developing country with serious economic problems [13].

Direct contact with intermediate domestic animals, or consumption of wild animals, was the initial route of transmission of SARS-CoV-2 to humans; however, the exact mechanism is unknown. Human-to-human infection is transmitted through large droplets generated during coughs and sneezes of symptomatic patients but can also occur in asymptomatic people in the incubation phase $[14,15]$. These infected droplets can spread from one to two meters and settle on surfaces. Virus can remain viable on surfaces for days in favorable atmospheric conditions but is destroyed in less than a minute with common disinfectants such as sodium hypochlorite, hydrogen peroxide, and others [16]. Virus is also present in feces and contaminating water supply and, according to one hypothesis, its subsequent transmission by aerosolization may occur. Patients can be infectious 
for as long as symptoms last, and even in clinical recovery, some people can act as super-transmitters depending on the number of people with whom they come in contact [17]. Studies have shown higher viral loads in the nasal cavity compared to the throat. There is no difference in viral load between symptomatic and asymptomatic people [18].

Its origin, high mutations and dissimilar manifestations in the host continue to be studied, as well as its rapid spread and viability both in temperate countries and in the tropics. The greatest circulation of the virus in Cuba with a tropical climate occurs in the summer months characterized by high temperatures and relative humidity. Recent studies have shown that Seasonal environmental factors modulate host airway immune responses and affect viability and transmission ways of respiratory viruses. Human behavior affects the contact rates between infected and susceptible individuals $[19,20]$. Virus is highly contagious causing respiratory symptoms such as pneumonia and severe respiratory distress syndrome in humans, also affecting the immune system, decreasing the white blood cells that are responsible for producing and secreting antibodies or specific immunoglobulins that help the body fight infections and other diseases [21].

Patients over 60 years of age and with chronic diseases are the most vulnerable, although all ages are susceptible. The most common symptoms are: fever, general weakness and a dry cough; severe cases are more likely to be older adults with multiple comorbidities. To date, there is no specific antiviral treatment; based on the current state, blocking transmission, isolation, respiratory and eye protection, and hand hygiene are the most effective strategies to prevent the spread of SARS-CoV-2. More studies are needed to determine the mechanism of pathogenicity, test antiviral drugs, and develop a vaccine to prevent new outbreaks of this disease [22].

\section{Dengue-SARS-CoV-2 Co-Circulation}

SARS-CoV-2 and dengue infections continue spreading, mainly in tropical settings causing a high impact on the health systems of the countries, where Cuba is not exempt from this problem. Recent studies show that dengue and SARS-CoV-2 co-circulation is a reality resulting to be more dangerous in regions that are endemic for arboviruses [2325]. Be able to warn the climatic conditions that favor the circulation of both viruses and their prediction, is one of the objectives of this multidisciplinary research.

It has been taken as a starting point, the identification that is known today about the climatic factors that may be influencing the viability, transmission and contagion of both viruses, SARS-CoV-2 by air from person to person, while dengue viruses use the Aedes Aegypti vector as a route of infestation (Figure1).

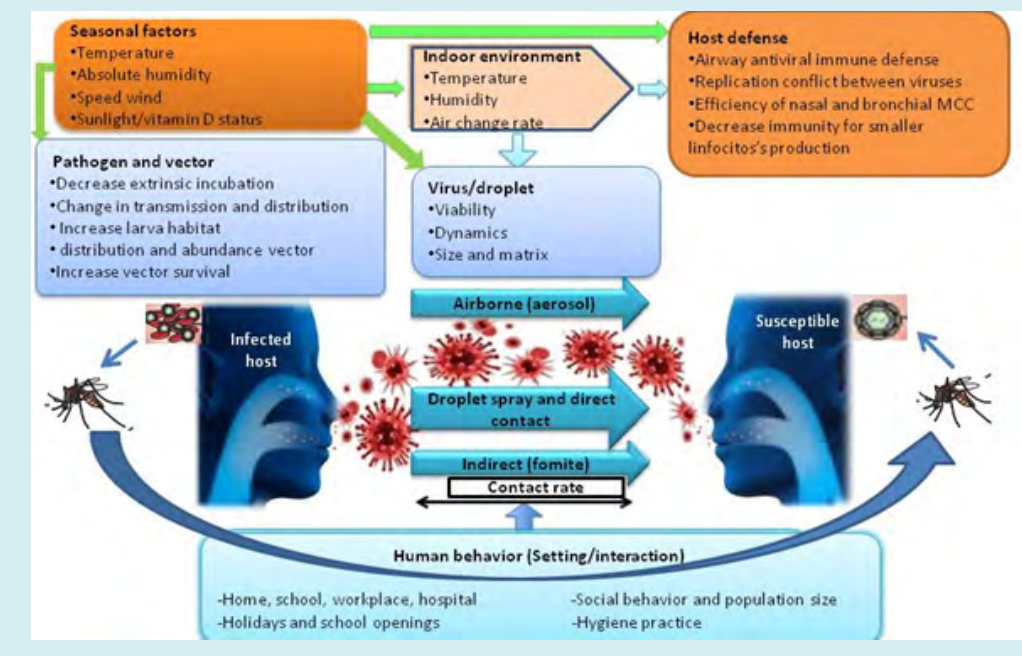

Figure 1: Effects of the environment and climate on Co-Circulation SARS-CoV-2 and dengue transmission. (Adapted from Moriyama M, et al. [19]).

This study makes it possible to improve the warning system with the purpose of: obtaining models for the prediction and early warning of the circulation of SARSCoV-2 virus and dengue transmitted by Aedes, from the climatic variability both on a temporal and spatial scale. In this sense, the working hypothesis is that both viruses could be circulating at the same time, causing the appearance of outbreaks in the same areas. Therefore, forecasting the viral dynamics based on the conditions that favor it, as well as the periods of greater host susceptibility associated with climate 


\section{Open Access Journal of Mycology \& Mycological Sciences}

variations, provides a tool for decision makers in the health system to take the necessary control measures. It also helps medical personnel make an early diagnosis according to the signs and symptoms that cause both diseases and that could be confused, if they do not know that both viruses are circulating at the same time and space.

\section{Materials and Methods}

Design: ecological study with retrospective-prospective analysis of the series of the SARS-CoV-2 viruses, dengue, as well as Ae focus with their index and the climatic anomalies described by the climatic Bulto Index (BI) in period 20102020. Complex climatic index $\left(\mathrm{BI}_{1, \mathrm{r} t \mathrm{t}}\right)$ developed by Ortiz were used $[1,6,8,26,27]$. The expression is generated from the analysis of time series of the climatic variables, applying techniques of multivariate analysis of principal components to generate the weights or contributions of the variables to each index, obtaining the orthogonal functions. Interpretation of $\left(\mathrm{BI}_{1, \mathrm{r}, \mathrm{t}}\right)$ describes inter-monthly and interseasonal variation; includes maximum and minimum mean temperature, precipitation, atmospheric pressure, vapor pressure, and relative humidity. $\left(\mathrm{BI}_{2, \mathrm{r}, \mathrm{t}}\right)$ describes seasonal and inter-annual variation; includes solar radiation and sunshine duration as factors that affect temperature and humidity. Positive values are associated with a high solar energy level.

\section{Statistical Methods}

Interpolation method for the spatial structure: to this analysis, a mesh with continuous information of 1200 nodes (Raster format) was generated. It means, transforming the information of a finite number of samples in a continuous space that allowed knowing the variation pattern in the area and comparing with those observed in the studied sample, as well as to characterize areas where information was not available. For that, the Kriging method combined with the method of the Inverse Distance (IDM) was implemented with the objective of interpolating, to have information in the whole study domain, with a resolution of $10 \mathrm{~km}^{2}$ [1]. The Multivariate Moran Index was used to determine the spatial correlation given by the relationship of the entomological variables and the climatic conditions described by $\left(\mathrm{BI}_{1, \mathrm{r}, \mathrm{t}}\right)[2]$.

For the prediction, the Simultaneous Autoregressive Models and the Spatial Autoregressive Conditional Models were used; The temporal modeling was carried out by using the Heteroscedastic Conditional Autoregressive and Autoregressive Models, both with exogenous variables. To obtain the co-circulation risk forecast maps, a stratification of the circulation of both viruses is carried out and map algebra is applied, with both information in raster format. Various adjustment tests were performed for predictive quality $[2,7,27-29]$.

For create multiannual graphics, the software Sigma Plot 10.0 style Filled Contour Plot (plots data values in 2D space filing in the area between contour levels) was used. The box plot and line plot of multiple variables graphics were performed using Statistical and Excel 2007. For statistical analysis the software S-Plus 2000, GS-plus 10.0 and SIG ArcGIS 10.1 were used. For processing of the series trends WinStat.V1.0 software was used.

\section{Results and Discussion}

Below are some preliminary results of this research that has allowed the prediction of both viruses, as well as their co-circulation.

\section{Dengue Prediction}

The high non-linear association between the seasonality of the climatic variability described through bioclimatic index $\left(\mathrm{BI}_{1, \mathrm{r}, \mathrm{t}}\right)$ the house and Breteau indexes and Dengue cases is shown. A delay or lag effect of one to two months is manifested, which allows describing the risks of Dengue circulation in the country, being potential predictors. The moments of greatest risk are the months corresponding to the second quarter of the rainy period August-October and the beginning of this (May) with very humid conditions (70-90\%), very high temperaturas $\left(29-35^{\circ} \mathrm{C}\right)$, high volume of precipitation (Annual precipitation is $1335 \mathrm{~mm}$ ), high cloudiness ( $>5 / 8$ cloudy sky) and low insolation $(<12$ light hours). These factors favor the presence of the vector and modify both its reproductive and feeding capacity, increasing the infestation with the different Dengue viruses.

In the transition periods (April and October) where sudden changes in climate variability occur, they could affect the response of the metabolic system that can result in oxidative stress and free radicals, bringing with it an immunosuppression, which favors the infections [30]. On the other hand, precipitation after several months with little rain usually results in an increase in the number of focus, leading to an outbreak.

The provinces were grouped according to their spatial climatic variation (According to $\left(\mathrm{BI}_{1, \mathrm{r}, \mathrm{t}}\right)$ takes values from negative anomalies $(-0.75 ;-3)$ of less rainy period November-April and positive anomalies $(0.75 ; 3)$ of rainy period May-October. April $(0 ;-0.75)$ and May $(0 ; 0.75)$ are transition months $[1,2]$ ) and the behavior of Dengue cases. The provinces of the Central-Eastern region are the ones with the highest presence of cases, except for Havana, which is grouped at the same level of risk associated with climatic variation. 
The utility provided by the prediction on a monthly scale, according to the Bulto indexes, is demonstrated well in advance. The forecast maps show the period of the year and the areas in the country where the climate impacts and favors the conditions for the increase of vector populations, the circulation of Dengue virus and an increase in cases (2.3-3.9\%). Although there is also an impact in terms of the increase of the affected areas and the displacement of the vector, which serve as a tool for early warning. This tool provides the current surveillance system with more time for prevention actions and decision-making by health authorities, before outbreaks of this disease occur.

\section{SARS-CoV-2 Prediction}

Heteroscedastic autoregressive and spatial autoregressive models using complex climatic indexes to simulate seasonal variability as exogenous variables are suitable for predicting the impact of climatic variability on the circulation of SARS-CoV-2. The quality of fit of the temporal model was determined with significant statistics with a high concordance and Skill factor of $0.95 \%$ and for the spatial model it was more than $0.90 \%$. A monthly early warning system of viral circulation in the country was obtained and it was determined which provinces have the highest viral circulation in different months of the year. The integrated and complex approach to climate allows a better understanding of the behavior mechanism of viruses and their association. When in the presence of high humidity $(>70 \%)$ and high temperatures $\left(>32^{\circ} \mathrm{C}\right)$, it is generally a response of the variable to a characterized climatic situation, which can be conditioned by the presence of a low-pressure system that leads to convection processes $[28,31]$.

Climatic conditions affect blood circulation, heart rhythm and respiration in the human body, since heat exchange is closely linked to the metabolic process, which is regulated by the nervous system. It can also weaken the respiratory system, interfering with the exchange of oxygen and carbon dioxide, which can lead to greater condensation and acidification in the respiratory ways, a very favorable condition for the virus multiplication [1,32].

All these results led to the creation of the dengue alert system and co-circulation with SARS-CoV-2 based on the observation and forecast of climatic factors, which strengthens the active health surveillance system (Figure 2).

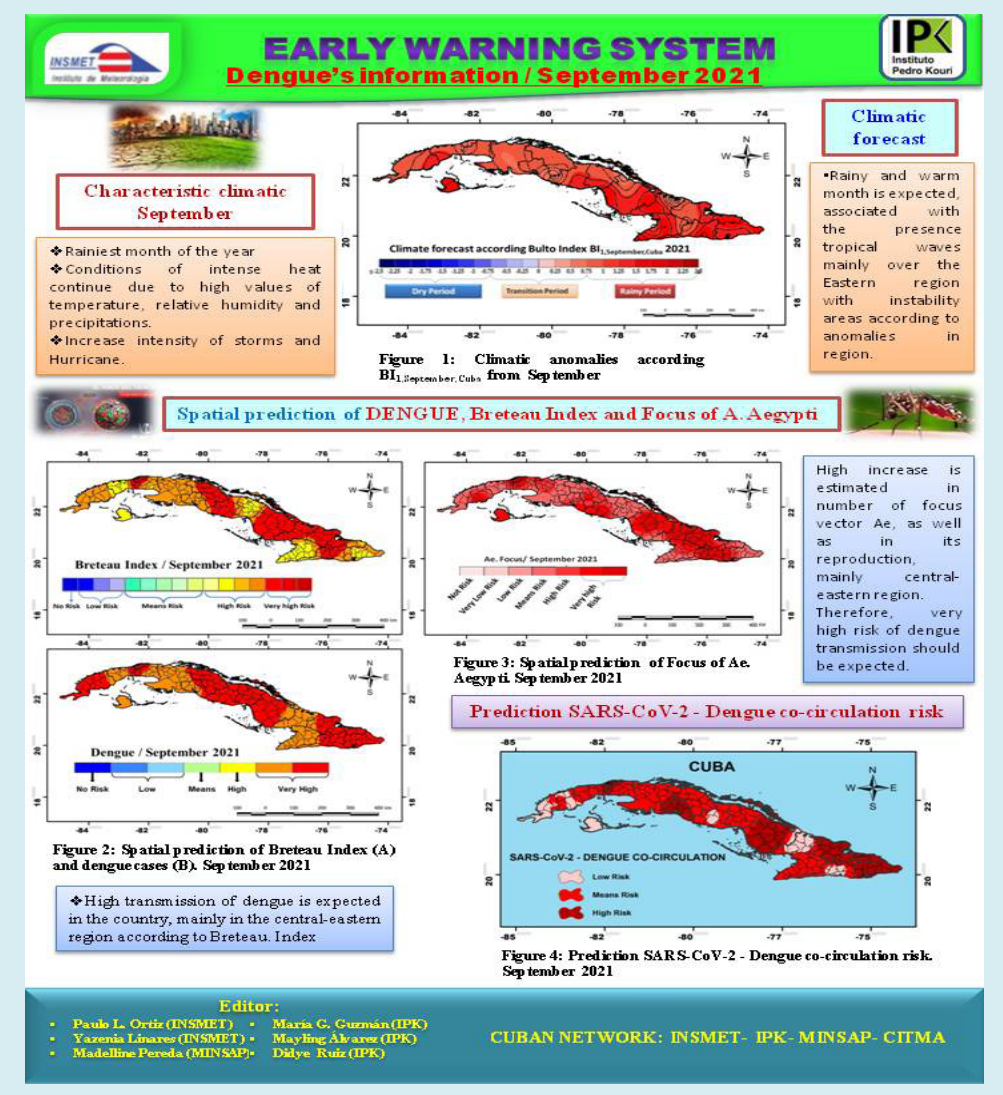

Figure 2: Early warm system of Dengue and co-circulation risk with SARS-CoV-2 from climate conditions in Cuba. 


\section{Open Access Journal of Mycology \& Mycological Sciences}

\section{Conclusions}

It is evident that the climate is an important determinant for the health sector, and in particular to understand the behavior and circulation of viruses, then any significant variation that climate experiences will bring changes in the load of microorganisms, vectors, reservoirs and susceptible human beings, generating changes in epidemiological patterns and entomological indicators, bringing with it an increased risk of dengue viruses circulation and then the report of disease cases in the country.

The results presented show that the epidemiological and entomological indicators and the dengue virus are susceptible to receiving the greatest impacts. The changes that are expected in the entomological index and therefore in the vector populations increases the risk of dengue appearance.

Both temporal and spatial models were obtained for the prediction and early warning of the circulation of SARS-CoV-2 and dengue viruses, conditioned by the impact of seasonal climate variability, that constitute a tool for early warning. This tool provides the current surveillance system with more time for prevention actions and decision-making by health authorities, before outbreaks occur.

\section{Research Perspectives}

The project intends to continue investigating the sensitivity of diseases and their causative agents to variability and climate change. It also aims to deepen the interactions with other ecological processes that help to understand the response mechanisms of infectious agents in relation to man. In addition to formulating forecast models based on integrated information (epidemiological, environmental, climatic and socioeconomic) from a complex approach, which allow the development of action plans in public health based on early warning systems that facilitate the identification of risk situations associated with climatic hazards.

Strengthen assessment and vulnerability activities to identify the contribution of climate variability and change to health, with an emphasis on infectious diseases. For this, the projections of the demographic structure of our country and the influence of other sectors in the different climate change scenarios are considered, as one of the objectives of the Cuban climate and health observatory.

\section{Acknowledgements}

The support by the Ministry of Science, Technology and Environment and the Ministry of Public Health is appreciated. Also thank the personnel of the network of meteorological stations of Institute Meteorology for their assistance in the processing of climatic data. Thanks to the medical and laboratory staff of the Pedro Kouri Institute of Tropical Medicine and microbiological laboratories of the country, for the contribution in obtaining the databases. Special recognition to the scientific members of the climatehealth team in research lines on arboviruses, respiratory viruses and climatology.

\section{References}

1. Vega YL, Ortiz PLB, Acosta BH, Valdés OR, Borroto SG, et al. (2018) Influenza's Response to Climatic Variability in the Tropical Climate: Case Study Cuba. Virology \& Mycology 7(2): 1000180.

2. Ortiz BPL, Perez A, Rivero A, Linares YV, Perez A, et al. (2015) Spatial models for forecasting and early warning behavior Aesdes Aeypti from climate variability and change in Cuba. MEDICC Review 17(2): 20-28.

3. Ortíz PL (2008) Assessment of Human Health Vulnerability in Cuba due to Climate or Weather Variability and Change. In book "Global Warming and Climate Change: Kyoto-Ten Years and Still Counting. Publisher Science, UK. 1-2.

4. Ortiz PL, Perez AR, Rivero AV, Leon NV, Diaz M, et al. (2006) Mini- Monograph Results of assessing the human health vulnerability to climate variability and change in Cuba. Environmental Health Perspectives 114(12): 1942-1949.

5. Vega YL, Ramirez OV, Herrera BA, Ortiz Bulto PL (2017) Impact of Climatic Variability in the Respiratory Syncytial Virus Pattern in Children Under 5 Years-Old Using the Bulto Climatic Index in Cuba. Int J Virol Infect Dis 2(1): 014-013.

6. Ortiz-Bulto PL, Vega YL, Ramírez OV, Herrera BA, Gutiérrez SB (2017) Temporal-spatial model to predict the activity of respiratory syncytial virus in children under 5 years old from climatic variability in Cuba. Int J Virol Infect Dis 2(1): 030-037.

7. Borroto S, Linares Y, Ortiz P, Valdés O, Acosta B (2020) Influence of climatic variability and respiratory viruses on the burden of medically attended acute respiratory infections in Cuba. J Respir Dis Med 2: 1-7.

8. Guzmán MG, Ortiz Bulto PL, Vega YL, Alberdi CP, Velazco VG, et al. (2020) Chapter 1: El virus SARS-CoV-2 y la covid-19. In: Noa RR, et al. (Eds.), "La Habana: Atlas de la covid-19”. Editorial UH, La Habana, Cuba, pp: 216.

9. Maria GG (2016) Dengue. Editorial Ciencias médicas. La 


\section{Open Access Journal of Mycology \& Mycological Sciences}

Habana, Cuba, pp: 512.

10. Siriyasatien P, Chadsuthi S, Jampachisri K, Kesorn K (2018) Dengue epidemics prediction: A survey of the State of the Art based on data Science processes. IEEE ACCESS 6: 53795.

11. Stewart-Ibarra AM, Muñoz AG, Ryan SJ, Ayala EB, BorborCordova MJ, et al. (2014) Spatiotemporal clustering, climate periodicity, and social-ecological risk factors for dengue during an outbreak in Machala, Ecuador, in 2010. BMC infectious diseases 14(1): 610.

12. Ryan SJ, Carlson CJ, Mordecai EA, Johnson LR (2019) Global expansion and redistribution of Aedes-borne virus transmission risk with climate change. PLoS Negl Trop Dis 13(3): e0007213.

13. (2021) Coronavirus in Cuba. MINSAP.

14. Chan JF, Yuan S, Kok KH, To KK, Chu H, Yang J, et al. (2020) A familial cluster of pneumonia associated with the 2019 novel coronavirus indicating person-toperson transmission: a study of a family cluster. Lancet 395(10223): 514-523.

15. Rothe C, Schunk M, Sothmann P (2020) Transmission of 2019-nCoV infection from an asymptomatic contact in Germany. N Engl J Med 382: 970-971.

16. Kampf G, Todt D, Pfaender S, Steinmann E (2020) Persistence of coronaviruses on inanimate surfaces and its inactivation with biocidal agents. J Hosp Infect 104(3): 246-251.

17. (2020) Coronavirus disease (COVID-19) Weekly Epidemiological Update and Weekly Operational Update. World Health Organization.

18. Zou L, Ruan F, Huang M (2020) SARS-CoV-2 viral load in upper respiratory specimens of infected patients. $\mathrm{N}$ Engl J Med 382: 1177-1179.

19. Moriyama M, Hugentobler WJ, Iwasaki A (2020) Annual Review of Virology Seasonality of Respiratory Viral Infections. Annual Rev Virol 7: 83-101.

20. Audi A, AlIbrahim M, Kaddoura M, Hijazi G, Yassine HM, et al. (2020) Seasonality of Respiratory Viral Infections: Will COVID-19 Follow Suit?. Front Public Health 8: 567184.

21. Abbas AK, Lichtman AH, Pillai S (2018) Cellular and molecular immunology. Elsevier 9: 576.

22. Bonilla OA (2020) Understanding the COVID-19 Medicent Electron 24(3).
23. Lopez PL, Lopez EM, Benavidez I (2020) COVID-19 in the dengue season. Rev Latin Infect Pediatr 33(3): 119-121.

24. Pan American Health Organization (2020) Control of Aedes aegypti in the scenario of simultaneous transmission of COVID-19. Washington

25. Costa J, Ferreira EC, Santos C (2021) COVID-19, Chikungunya, Dengue and Zika Diseases: An Analytical Platform Based on MALDI-TOF MS, IR Spectroscopy and RT-qPCR for Accurate Diagnosis and Accelerate Epidemics Control. Microorganisms 9(4): 708.

26. (2013) Impacto del Cambio Climático y Medidas de Adaptación en Cuba. INSMET, pp: 520.

27. Arens MQ, Buller RS, Rankin A, Mason S, Whetsell A, et al. (2010) Comparison of the Eragen Multi-Code Respiratory Virus Panel with conventional viral testing and real-time multiplex PCR assays for detection of respiratory viruses. J Clin Microbiol 48(7): 2387-2395.

28. Linares-Vega Y, Ortiz-Bultó PL, Borroto-Gutiérrez S, Acosta-Herrera B, Valdés-Ramírez O, et al. (2020) Modeling and Predicting the Impact of Climate Variability on Influenza Virus Spread in Cuba. J Mycol Mycological Sci 3(3): 000130.

29. Tompkins AM, LoweR, Nissan H, Martiny N, Roucou $P$, et al. (2019) Chapter22: Predicting Climate Impacts on Health at Sub-seasonal to Seasonal Timescales. Subseasonal to Seasonal Prediction, pp: 455-477.

30. Ramnath V, Rekha P, Sujatha K (2008) Amelioration of heat stress induced disturbances of antioxidant defense system in chicken by Brahma Rasayana. Evid Based Complement Alternat Med 5(1): 77-84.

31. Lecha EL, Paz RL, Lapinel PB (1994) The climate of Cuba. Editorial Academia. La Habana, Cuba, pp: 186.

32. Ishmatov A (2017) On the connection between supersaturation in the upper airways and «humidrainy» and «cold-dry» seasonal patterns of influenza. Peer J Preprint pp: 1-14. 